\title{
A randomised study of the effects of letrozole and anastrozole on oestrogen receptor positive breast cancers in postmenopausal women
}

\author{
J. Murray $\cdot$ O. E. Young $\cdot$ L. Renshaw $\cdot$ S. White $\cdot$ \\ L. Williams · D. B. Evans · J. St. J. Thomas • \\ M. Dowsett · J. M. Dixon
}

Received: 11 April 2008/Accepted: 11 April 2008/Published online: 26 April 2008

(C) Springer Science+Business Media, LLC. 2008

\begin{abstract}
Introduction Changes in proliferation as measured by Ki67 occur within 14 days of starting treatment with an aromatase inhibitor and these changes have been shown to be predictors of long term outcome. This study aimed to compare changes in proliferation following 14 days of treatment with anastrozole and letrozole. Methods Two hundred and six women with 209 estrogen receptor (ER) positive operable breast cancers (three bilateral) were randomly allocated to receive either 14 days treatment with $2.5 \mathrm{mg}$ of letrozole or $1 \mathrm{mg}$ of anastrozole prior to surgery. Changes in expression of estrogen (ER) and progesterone receptors (PgR) as assessed by ALLRED scores and proliferation as assessed by Ki67 were analysed. The HER2 status of each tumour was also assessed using a combination of the Hercept test and FISH. Results Both letrozole and anastrozole reduced ER expression (ALLRED score) by a mean
\end{abstract}

Electronic supplementary material The online version of this article (doi:10.1007/s10549-008-0027-0) contains supplementary material, which is available to authorized users.

J. Murray · O. E. Young $\cdot$ L. Renshaw $\cdot$ S. White

J. St. J. Thomas · J. M. Dixon ( $\square)$

Edinburgh Breast Unit, Western General Hospital,

Edinburgh EH4 2XU, UK

e-mail: jmd@ed.ac.uk

L. Williams

Medical Statistics Unit, University of Edinburgh,

Medical School, Edinburgh, UK

D. B. Evans

Oncology Research, Novartis Institutes for BioMedical Research Basel, 4002 Basel, Switzerland

M. Dowsett

Academic Biochemistry, Royal Marsden Hospital,

London SW3 6JJ, UK of $0.32(0.20-0.44), P<0.001$ and $P g R$ fell by a mean of 2.54 (2.20-2.89) $P<0.0001$. Letrozole reduced proliferation from a geometric mean of $6.37 \%$ to $0.81 \%, P<0.0001$ and anastrozole reduced proliferation from $5.81 \%$ to $0.77 \%$, $P<0.0001$. There was no differences between drugs in the fall in ER, PgR or proliferation. Both letrozole and anastrozole produced significant falls in proliferation in both HER2 positive and HER2 negative cancers, all $P<0.001$. Discussion 14 days of both letrozole and anastrozole reduces proliferation, ER and $\mathrm{PgR}$ expression. No significant difference between these two drugs was identified.

Keywords Aromatase inhibitors - Breast cancer · Postmenopausal · Oestrogen receptor

\section{Introduction}

Approximately $75 \%$ of human breast cancers express oestrogen receptor (ER) and are considered ER positive [1]. In these tumours oestrogen deprivation or antagonism are effective treatments for some but not all women. The two non-steroidal third generation aromatase inhibitors anastrozole and letrozole have been shown to be more effective than tamoxifen in postmenopausal women with ER positive breast cancers in the metastatic, neoadjuvant and adjuvant settings [2-8]. In the PO24 neoadjuvant study, letrozole was statistically superior to tamoxifen in all endpoints [4], whereas in the IMPACT study neoadjuvant anastrozole produced similar response rates to tamoxifen although there were no significant differences in clinical and ultrasound objective response rates between the three arms, but there was a significantly greater rate of breast conserving surgery in women randomised to receive anastrozole compared to tamoxifen, $P=0.03$ [3]. 
In the IMPACT study, measurement of Ki67 after 2 weeks of treatment was predictive of the efficacy of these drugs in the parallel adjuvant ATAC trial [9]. Additionally Ki67 levels after 2 weeks of treatment correlated significantly with relapse free survival in this pre-surgical study [10]. These findings support the validity of short term changes in $\mathrm{Ki} 67$ as an intermediate marker of clinical effectiveness of endocrine therapy [11, 12]. This justifies the selection of 2 weeks treatment duration of the current study.

There have been few trials comparing the efficacy of anastrozole and letrozole directly. One study did compare the two drugs as second line therapy in advanced breast cancer and showed no difference in the primary endpoint of time to progression [13]. Studies comparing the potency of these two agents in reducing circulating oestrogen have shown that letrozole is a more complete inhibitor of aromatase than anastrozole $[13,14]$.

The aim of this study was to compare whether there was a significant difference between letrozole and anastrozole in their short term effects on ER, PgR and proliferation in a series of postmenopausal women with ER positive operable breast cancers. Secondarily the study set out to investigate the effects of these two agents in subsets of HER2 positive and HER2 negative cancers because it has been hypothesised that aromatase inhibitors may be particularly effective in the subset of patients that are HER2 positive.

\section{Patients and methods}

All patients with suspected breast cancer in Edinburgh have a core biopsy performed at the initial clinic visit using an automated 14 gauge hand held biopsy gun which is usually image guided. The aim is to get 4-5 good cores for histology assessment.

Two hundred and eleven patients were recruited into the study. Eligibility criteria for this study included histologically confirmed invasive cancer which was ER positive (ALLRED score of 2 or more) in women who were postmenopausal, as defined by no menstruation over the last 12 months and/or FSH/LH levels being within the postmenopausal range. Eligibility criteria excluded patients who were taking hormones or other treatments during the study period. Patients who had previously taken HRT were included provided they had stopped HRT at least 2 weeks prior to trial entry.

After giving informed written consent, patients were randomised to receive 14 days of treatment with anastrozole $(1 \mathrm{mg})$ or 14 days of treatment with letrozole ( $2.5 \mathrm{mg}$ ). The study was open labelled and was approved by the Lothian Research Ethics Committee. There were five major protocol violations; three patients entered but did not take any tablets; one patient did not proceed to surgery (as the patient was diagnosed with bone metastases) and one patient was premenopausal on hormone analysis. These five were not included in any analyses. Two hundred and six patients with 209 operable ER positive breast cancers completed the study which ran between July 2002 and August 2003.

Patients underwent surgery 14 days after starting letrozole or anastrozole and took their last tablet on the day of surgery. Fixed tissue was available from all patients from the diagnostic biopsy and from the surgical excision specimen.

After operation post-operative adjuvant therapy was based on the histology, stage and hormone receptor status and did not differ from patients not in this trial. Relatively few patients received adjuvant aromatase inhibitors as tamoxifen was the standard hormonal agent in use in 20022003 in the unit. All patients within this study are under follow up and data on disease free survival and overall survival is being collected with the aim of correlating 14 day Ki67 changes and outcome.

\section{Power calculation}

All eligible patients presenting to the Edinburgh Breast Unit between July 2002 and August 2003 were approached for inclusion in this trial. To detect a significant difference between drugs with 200 patients the baseline Ki67 would have to be reduced by $84 \%$ (relative ratio 6.1 ) on one drug and by $90 \%$ (relative ratio 9.9 ) on the other drug.

\section{Analysis of markers}

Fixed tissue from initial core biopsy and the excised tumour were stained in the Department of Pathology and scored by two independent observers. The pre and post surgery samples were stained in the same run and a range of positive and negative controls were included in each run.

\section{ER expression}

ER $\alpha$ expression was assessed within the Edinburgh Breast Unit laboratory of the University of Edinburgh using the primary antibody (ER $\alpha$ clone 6F11 from mouse melanoma p3 ms1-Ag4-1 supplied by Novacastra) applied at a concentration of 1 in 25 .

PgR expression

The primary antibody was the anti PgR antibody (Clone PgR 636) supplied by Dako cytomation used at a concentration of 1 in 100 . 
Ki67 proliferation associated antigen expression

The primary antibody was an MIB1 anti-Ki67 antibody supplied by DAKO cytomation and used at a concentration of 1 in 50 . Ki67 expression was recorded as the percentage of positively staining nuclei: two observers each counted at least 1,000 cells and the mean value of the two scores was used.

\section{HER2 expression}

Immunohistochemistry using the HercepTest (DAKO) was performed and scored according to the manufacturer's recommendations in a reference laboratory (the Department of Academic Biochemistry in the Royal Marsden Hospital, London). Manual scores of 2+ were tested for amplification by fluorescence in situ hybridization (FISH). The Pathvysion HER-2 FISH kit was used according to manufacturer's recommendations and results with an amplification ratio of $\geq 2.0$ were accepted as positive.

\section{Statistical analysis}

The statistical analysis was conducted by an independent statistician. All 206 patients were included in the analysis. The hormone therapy for each patient was coded to maintain the blind assessment and avoid bias. Proliferation scores were transformed on the log scale to achieve Normality of the data. Results are give as the geometric mean and confidence intervals, in order to preserve the units of measurement, although the analysis was conducted on the transformed data.

Analysis of variance was the principal method of analysis, supported by the $\chi^{2}$ test for trend for discrete data. Fisher's Exact test was used to examine the relationship between drug and number of patients for whom proliferation had fallen to below $1 \%$ after 14 days.

\section{Results}

One hundred and one patients received anastrozole (103 tumours) and 105 received letrozole (106 tumours). Two patients had 28-35 days of treatment due to surgery being postponed for health reasons. Nine patients had minor protocol violations taking drugs for slightly fewer or longer than 14 days: one patient (12 days); two patients (13 days); five patients (15 days); one patient (18 days).

The average age of the patients enrolled in the study was 64 years with a range of 43-86. Average tumour size (as measured by ultrasound) was $19.6 \mathrm{~mm}$ with a range of 3-70 $\mathrm{mm}$. The patients had an average Nottingham Prognostic Index of 3.8 with a range of 2.1-7.4. One patient in the anastrozole group had insufficient invasive cancer in the core biopsy when re-cut for the study and so the total number in the anastrozole arm available for analysis was 100 patients with 102 cancers.

\section{Changes in ER expression}

There was a mean fall in ER Allred score in the whole series of 0.32 (95\% CI [0.20, 0.44] $P<0.0001)$. The degree of fall in ER in individual patients treated with anastrozole and letrozole is shown in Table 1. There was a mean fall of 0.23 [9.06, 0.40], $P=0.0033$ for anastrozole and a mean fall of $0.41[0.24,0.57], P<0.0001$ for letrozole. The mean difference between the mean falls (anastrozole-letrozole) was $0.18[-0.42,0.05], P=0.14$, indicating no differences between the two drugs.

\section{Changes in $\mathrm{PgR}$ receptor}

There was a mean fall in the progesterone receptor Allred score in the whole series of 2.54 (95\% CI [2.20, 2.89]) $P<0.0001$. The changes in individual patients for the two drugs are shown in Table 2 (see supplementary material).

Anastrozole treatment resulted in a mean fall of 2.36 [1.87, 2.86], $P<0.0001$ and letrozole a mean fall of 2.72 [2.23, 3.20], $P<0.0001$. There was no significant difference between the drugs (mean difference (anastrozoleletrozole $)=-0.35[-1.05,0.34], P=0.32)$. Sixty-five out of $102(64 \%)$ had a reduction in $\mathrm{PgR}$ on anastrozole compared with 75 of $106(71 \%)$ on letrozole $(P=0.3)$.

\section{Ki67 status}

In 200 of the 208 cancers there was a fall in the percentage of cells staining positive with Ki67 antibody following treatment. The changes in Ki67 with both anastrozole and letrozole were highly significant for both drugs and are shown in Table $3 \mathrm{a}$.

Table 1 Change in Allred ER score after 14 days treatment with letrozole or anastrozole

\begin{tabular}{llc}
\hline ER score following treatment & Anastrozole & Letrozole \\
\hline 4 Units lower & 1 & 3 \\
3 Units lower & 0 & 1 \\
2 Units lower & 2 & 3 \\
1 Unit lower & 27 & 34 \\
No change & 60 & 53 \\
1 Unit higher & 12 & 12 \\
& $\chi^{2}$ for trend, $P=0.13$
\end{tabular}

$n$, number of tumours 
Table 3a Changes in percentage of cells staining positively for Ki67 after 14 days treatment with letrozole or anastrozole

\begin{tabular}{lccc}
\hline & Anastrozole & Letrozole & $\begin{array}{c}P \text {-value of differences } \\
\text { between drugs }\end{array}$ \\
\hline Baseline $(\%)$ & $5.81(4.94,6.82)$ & $6.37(5.42,7.49)$ & 0.44 \\
Post-treatment (\%) & $0.77(0.59,1.00)$ & $0.81(0.62,1.04)$ & 0.80 \\
Relative change: baseline/post-treatment ${ }^{\mathrm{a}}$ & $6.11(4.80,7.79)$ & $6.44(5.05,8.19)$ & 0.79 \\
Change from baseline $P$-value & $<0.0001$ & $<0.0001$ & \\
\hline
\end{tabular}

Values are given as the back-transformed geometric means $(95 \% \mathrm{CI})$ in order to preserve the units of measurement, although $P$-values were calculated using the log transformed data

${ }^{\text {a }}$ Relative size of baseline to post-treatment percentages

Table 3b Number of tumours with Ki67 $<1 \%$ after treatment

\begin{tabular}{lll}
\hline & Anastrozole & Letrozole \\
\hline Ki67 $\leq 1 \%$ & 58 & 58 \\
Ki67 $>1 \%$ & 44 & 48 \\
\hline
\end{tabular}

There was no significant difference in the changes in Ki67 between anastrozole and letrozole, $P=0.79$ and no difference between drugs in the numbers of tumours in which proliferation was reduced to less than $1 \%$ (Table 3b).

Patients with ER scores of 6-8 had lower initial rates of proliferation than patients who had ER scores of ER 2-5 $(P=0.04)$. The differences in the fall in proliferation between ER poor (2-5) and ER rich (6-8) cancers are presented in Table 4a. There was a statistically significant difference between ER categories in the numbers of tumours after treatment having $1 \%$ or less Ki67 positive cells, with tumours having ER scores of 6-8 being significantly more likely to have Ki67 scores of less than or equal to $1 \%$ at 14 days post treatment compared with tumours with an Allred score of $2-5 \quad(P=0.008)$ (Table 4b).

The changes in Ki67 in both ER poor (2-5) and ER rich (6-8) were statistically significant for both drugs although the changes were not significant between the two drugs (Table 5a and b). Only four tumours had ER scores of two, and of these four, two fell to ER zero, one remained the
Table 4b Number of tumours with Ki67 $<1 \%$ after treatment

\begin{tabular}{lcc}
\hline & ER poor & ER rich \\
\hline Ki67 $\leq 1 \%$ & 5 & 111 \\
Ki67 $>1 \%$ & 14 & 78 \\
\hline
\end{tabular}

same and one increased to ER3. However, the relative ratio in proliferation for these four tumours was 13.9 (92\% fall from baseline to post-treatment), with a confidence interval of $4.4-43.9(22.7 \%, 97.7 \%), P<0.0001$.

The effects of letrozole and anastrozole on proliferation were not significantly influenced by $\mathrm{PgR}$. The relative ratio in Ki67 in the PgR positive group $(n=158)$ was 7.39 $(5.61,9.72)$ (geometric mean and $95 \% \mathrm{CI}$ ) with anastrozole and $6.75(5.23,8.71)$ with letrozole (both ratios $P<0.0001)$. In PgR negative patients $(n=50)$ the falls were $4.18(2.66,6.56)$ and $5.87(3.52,9.77)$ respectively (both ratios $P<0.0001$ ). The difference in ratios in Ki67 between $\mathrm{PgR}$ positive and negative groups almost reached statistical significance at the 5\% level $(P=0.054)$, but the sample is likely to be underpowered to detect a significant difference in this dimension.

\section{HER2 Status}

Tumours that were HER2 positive had a significantly higher initial proliferation than tumours that were HER2 negative $(P=0.03)$ (Table 6a). There were marked

Table 4a Reduction in proliferation by ER status: ER poor (ER2-5), ER rich (ER6-8)

\begin{tabular}{lccc}
\hline & ER poor $(n=19)$ & ER rich $(n=189)$ & $\begin{array}{c}P \text {-value of differences } \\
\text { between ER status }\end{array}$ \\
\hline Baseline $(\%)$ & $8.58(5.97,12.28)$ & $5.87(5.20,6.62)$ & 0.04 \\
Post-treatment $(\%)$ & $1.83(1.05,3.11)$ & $0.72(0.59,0.87)$ & 0.002 \\
Relative change: baseline/post-treatment ${ }^{\mathrm{a}}$ & $4.17(2.38,7.20)$ & $6.57(5.48,7.88)$ & 0.13 \\
Change from baseline $P$-value & $<0.0001$ & $<0.0001$ & \\
\hline
\end{tabular}

Values are given as the back-transformed geometric means $(95 \% \mathrm{CI})$ in order to preserve the units of measurement, although $P$-values were calculated using the log transformed data

${ }^{\text {a }}$ Relative size of baseline to post-treatment percentages 
Table 5 (a) Ki67 changes with anastrozole in ER poor (2-5) and rich (6-8) tumours. (b) Changes in Ki67 with letrozole in ER poor (2-5) and rich (6-8) tumours

$$
\text { ER poor }(n=8) \quad \text { ER rich }(n=94) \quad P \text {-value of differences }
$$
between ER status

(a)

$\begin{array}{llll}\text { Baseline }(\%) & 8.15(4.72,13.96) & 5.63(4.78,6.62) & 0.21 \\ \text { Post-treatment }(\%) & 1.94(0.83,4.27) & 0.71(0.53,0.92) & 0.03 \\ \text { Relative change: baseline/post-treatment }^{\mathrm{a}} & 3.76(1.61,8.44) & 6.37(5.00,8.11) & 0.22 \\ \text { Change from baseline } P \text {-value } & 0.0009 & <0.0001 & \end{array}$

Change from baseline $P$-value

ER poor $(n=11)$

ER rich $(n=95)$

$P$-value of differences between ER status

(b)

Baseline (\%)

Post-treatment (\%)

Relative change: baseline/post-treatment ${ }^{\mathrm{a}}$

Change from baseline $P$-value
$8.94(5.40,14.70)$
$1.77(0.82,3.62)$
$4.48(2.08,9.45)$
$<0.0001$

$\begin{array}{ll}6.05(5.05,7.26) & 0.17 \\ 0.73(0.56,0.95) & 0.035 \\ 6.71(5.16,8.71) & 0.32\end{array}$

$<0.0001$

Values are given as the back-transformed geometric means $(95 \% \mathrm{CI})$ in order to preserve the units of measurement, although $P$-values were calculated using the log transformed data

${ }^{a}$ Relative size of baseline to post-treatment percentages

reductions in Ki67 in both the HER2+ve and HER2-ve cancers following treatment (Table 6a). Both anastrozole and letrozole reduced proliferation significantly in both HER2+ve and HER2-ve groups $(P<0.0001$ for all groups).

Patients who had HER2+ve cancers had a lower initial PgR score than those with HER2-ve cancers (HER2+ve $n=24$, mean PgR at diagnosis 3.29 (2.12-4.46); HER2-ve $n=184$, mean PgR at diagnosis 4.67 (4.25, 5.10); difference HER2-ve - HER2+ve $=1.38 \quad(0.14-2.63) \quad P=0.03)$. There was no evidence of HER2 status influencing the fall in $\operatorname{PgR}(P=0.15)$ after drug treatment, or of any interaction between HER2 status and PgR status (Table 6b).

\section{Discussion}

Anastrozole and letrozole are potent third generation aromatase inhibitors which cause profound depression of plasma oestrogen levels in postmenopausal women [15]. There is evidence that there are differences between the two drugs with letrozole being a more potent inhibitor of aromatase [16] and at clinically used doses letrozole reduces oestrogen levels to a greater degree than anastrozole [14]. There is some indirect evidence suggesting that there may be a dose response relationship between the degree of oestrogen suppression and the clinical effectiveness of drugs in breast cancer. One large

Table 6 (a) Reduction in proliferation (Ki67) in relation to HER2 status. (b) Reduction in proliferation (Ki67) in relation to HER2 status and PgR status

HER2-ve $(n=184) \quad$ HER2+ve $(n=24) \quad P$-value of differences
between HER2 status

(a)

Baseline (\%)

Post-treatment (\%)

Relative change: baseline/post-treatment ${ }^{\mathrm{a}}$

Change from baseline $P$-value

(b)

Relative change in $\operatorname{PgR}-$ ve patients $(n=50)$

Relative change in $\mathrm{PgR}+$ ve patients $(\mathrm{n}=158)$

$P$-value of differences between PgR status

$$
\begin{aligned}
& 5.81(5.14,6.56) \\
& 0.75(0.62,0.91) \\
& 6.24(5.20,7.48) \\
&<0.0001
\end{aligned}
$$

$$
\begin{aligned}
& 4.78(3.32,6.89) n=41 \\
& 6.97(5.73,8.47) n=143 \\
& 0.08
\end{aligned}
$$

$\begin{array}{ll}8.67(2.60,27.93) & 0.03 \\ 1.09(0.61,1.84) & 0.20 \\ 6.78(4.17,10.95) & 0.75\end{array}$

$<0.0001$

$\begin{array}{ll}5.22(2.40,11.39) n=9 & 0.84 \\ 8.25(4.51,15.07) n=15 & 0.60 \\ 0.36 & \end{array}$

Values are given as the back-transformed geometric means $(95 \% \mathrm{CI})$ in order to preserve the units of measurement, although $P$-values were calculated using the log transformed data

${ }^{\text {a }}$ Relative size of baseline to post-treatment percentages 
randomised trial demonstrated greater efficacy of $2.5 \mathrm{mg}$ of letrozole which produces $99 \%$ aromatase inhibition compared with $500 \mathrm{mg}$ of aminoglutethimide which inhibits aromatase by approximately $85 \%$ indicating that more potent inhibitors of aromatase may produce a better clinical outcome [17].

Clinical data indicate that both letrozole and anastrozole are effective agents in postmenopausal women with ER positive breast cancer. In the first line study comparing letrozole and tamoxifen in patients with metastatic breast cancer, letrozole was superior in all endpoints both in the intention to treat and in subgroup analysis [2]. While anastrozole has been shown to be superior to tamoxifen in postmenopausal women with breast cancer in the subgroup of patients known to be ER positive, no significant benefit was seen in the intention to treat analysis [18]. In the neoadjuvant setting letrozole was again superior to tamoxifen in all endpoints [4] whereas studies comparing anastrozole and tamoxifen showed no significant improvement in response rates with anastrozole although greater numbers of patients did achieve breast conserving surgery with anastrozole than with tamoxifen [3].

There is one direct study comparing anastrozole and letrozole in the second line setting in patients with metastatic disease who had already received tamoxifen. There was no difference in the primary endpoint of time to progression but a significantly greater overall response rate was reported in patients treated by letrozole compared to women treated by anastrozole [13]. This improved response rate was only seen in patients whose ER status was unknown. In patients whose cancer was known to be ER positive there was no apparent difference in the response rate between the two drugs.

There is increasing interest in identifying biological markers that will allow direct comparisons of drug efficacy. Ki67 has been studied extensively and has been widely used both as a prognostic marker and as a biological marker to compare drug effects [19]. In the IMPACT study the efficacy of anastrozole alone, tamoxifen alone or the two drugs together in reducing Ki67 paralleled their efficacy in the adjuvant ATAC trial [3]. The changes in Ki67 in patients treated with differing doses of fulvestrant also mirrored clinical efficacy with doses of $250 \mathrm{mg}$ producing greater reductions in Ki67 and being more effective clinically than doses of $125 \mathrm{mg}$. Higher doses of fulvestrant were also more effective at down regulating both ER and PgR expression [20]. More recently data from the IMPACT trial have shown that the absolute level of Ki67 after 2 weeks of treatment is an independent predictor for disease free survival [10]. There are thus ample data to indicate that changes in Ki67 during treatment with endocrine agents offer an opportunity to evaluate and compare the effectiveness of different drugs.
Although there was a small fall in ER in patients treated by 14 days of either anastrozole or letrozole, the majority of patients had either no fall or only a change of one Allred score. This fall in ER is almost certainly explained by the variation previously described when comparing cores and tissue sections [21], and importantly in a parallel neoadjuvant study in which patients had a core biopsy at diagnosis and after 2 weeks treatment no consistent fall in ER was seen [22]. Recently Ellis did report that breast cancers which lost their ER expression at 4 months had a poor long term outcome [23]. Whether the falls in ER observed in this study indicate some cancers losing hormone independence is not clear. There were significant falls in PgR following treatment and the majority of patients treated with anastrozole and letrozole had reductions in PgR expression. Although the mean fall was higher with letrozole $2.72(2.23-3.20)$ than anastrozole 2.36 (1.87-2.86), the difference between the two drugs was not statistically significant, $P=0.32$. The failure to identify significant differences between the two drugs suggests either that the significant differences in the degree of oestrogen suppression between the two agents is not associated with significant differences in their ability to down regulate PgR expression or that insufficient numbers were included in this study to detect any small difference that does exist between the two drugs.

Both letrozole and anastrozole were extremely effective at reducing proliferation with no evident difference between the two drugs. Of 208 cancers, there was a fall in proliferation in 200. Both drugs were effective in all ER categories. Significantly greater numbers of patients with ER rich cancers (Allred scores 6-8) compared with ER poor cancers (2-5) achieved a cell cycle response and switch off proliferation $(<1 \%+v e$ cells). Tumours with an ER score of 2 have previously been considered as ER negative and were included in this study to explore whether aromatase inhibitors did produce significant biological effects in these cancers. The decision to classify these tumours as ER negative was based on work with tamoxifen but in this study both aromatase inhibitors produced falls in proliferation in such cancers questioning the current classification of these tumours as ER negative.

HER2 positive cancers (14\% of total group) had a significantly higher initial proliferation rate and a lower PgR level than HER2 negative cancers. There were significant falls in proliferation in both HER2 positive and HER2 negative cancers with both aromatase inhibitors with no evidence to suggest differences between HER2 negative and HER 2 positive cancers in terms of percentage Ki67 decrease. These results support clinical observations that response rates to letrozole in the neoadjuvant setting are similar for both HER2 positive and HER2 negative cancers [24]. Although the degree of reduction in proliferation was 
similar in HER2 negative and HER2 positive cancers, the 14 day $\mathrm{Ki} 67$ in the HER2 positive cancers was higher $(1.09 \%(0.61,1.84))$ than HER2 negative cancers $(0.75 \%$ $(0.62,0.91))$. This is consistent with our recent observation that greater residual proliferative activity remains in HER2 positive cancers following treatment with aromatase inhibitors [24]. If what matters is residual activity rather than absolute reduction in proliferation then this might indicate a degree of aromatase inhibitor resistance in HER2 positive cancers.

A major and rapid reduction in proliferation is seen in the majority of ER positive cancers with both anastrozole and letrozole. No difference in any biological marker was identified between these two agents but a further study comparing these two drugs in ER poor cancers is underway. The Femara Anastrozole Clinical Evaluation (FACE) study, a large, phase IIIb trial comparing adjuvant letrozole versus anastrozole in postmenopausal women with node-positive, ER+ breast cancer will be needed to determine whether there is a difference in the clinical differences of these two agents.

\section{References}

1. Barnes DM, Millis RR, Billett CE et al (2004) The interaction of oestrogen receptor status and pathological features with adjuvant treatment in relation to survival in patients with operable breast cancer: a retrospective study of 2660 patients. Endocr Relat Cancer 11:85-96

2. Mourisden H, Gershanovich M, Sun Y et al (2001) Superior efficacy of letrozole versus tamoxifen as first-line therapy for postmenopausal women with advanced breast cancer: results of a phase III study of the International Letrozole Breast Cancer Group. J Clin Oncol 19:2596-2606

3. Smith IE, Dowsett M, Ebbs SR et al (2005) Neoadjuvant treatment of postmenopausal breast cancer with anastrozole, tamoxifen, or both in combination: the Immediate Preoperative Anastrozole, Tamoxifen or Combined with Tamoxifen (IMPACT) multicenter double-blind randomized trial. J Clin Oncol 23:5108-5116

4. Eiermann W, Paeple S, Appfelstaedt J et al (2001) Preoperative treatment of postmenopausal breast cancer patients with letrozole: a randomized double-blind multicenter study. Ann Oncol 12:1527-1532

5. Bonneterre J, Thurlimann B, Robertson JF et al (2000) Anastrozole versus tamoxifen as first-line therapy for advanced breast cancer in 668 postmenopausal women: results of the Tamoxifen or Arimidex Randomized Group Efficacy and Tolerability study. J Clin Oncol 18:3748-3757

6. Vergote I, Bonneterre J, Thurlimann B et al (2000) Randomised study of anastrozole versus tamoxifen as first-line therapy for advanced breast cancer in postmenopausal women. Eur J Cancer 36(suppl 4):S84-S85

7. Howell A, Cuzick J, Baum M et al (2005) Results of the ATAC (Arimidex, Tamoxifen, Alone or in Combination) trial after completion of 5 years' adjuvant treatment for breast cancer. Lancet 365:60-62

8. Coates AS, Keshaviah A, Thurlimann B et al (2007) Five years of letrozole compared with tamoxifen as initial adjuvant therapy for postmenopausal women with endocrine-responsive early breast cancer: update of study BIG 1-98. J Clin Oncol 25:486-492

9. Dowsett M, Ebbs SR, Dixon JM et al (2005) Biomarker changes during neoadjuvant anastrozole, tamoxifen, or the combination: influence of hormonal status and HER-2 in breast cancer-a study from the IMPACT trialists. J Clin Oncol 23:2477-2492

10. Dowsett M, Smith IE, Ebbs SR et al (2005) Short-term changes in Ki-67 during neoadjuvant treatment of primary breast cancer with anastrozole or tamoxifen alone or combined correlate with recurrence-free survival. Clin Cancer Res 11:951s-958s

11. Murray E, Renshaw L, Macaskill EJ et al (2006) Factors predicting survival after neoadjuvant therapy with aromatase inhibitors. Breast Cancer Res Treat 100:S23

12. Llombart A, Ruiz A, Lopez-Guerrero JA et al (2006) Objective response to neoadjuvant letrozole predicts distant disease free survival in post-menopausal women with stage ii-iii $\mathrm{ER} / \mathrm{PgR}$ positive breast cancer. Ann Oncol 17(suppl 9):ix96

13. Geisler J, Haynes B, Anker G (2002) Influence of letrozole and anastrozole on total body aromatization and plasma estrogen levels in postmenopausal breast cancer evaluated in a randomized, cross-over study. J Clin Oncol 20:751-757

14. Dixon JM, Renshaw L, Young O, Murray J, Macaskill EJ, McHugh M, Folkerd E, Cameron DA, A'Hern RP, Dowsett M (2008) Letrozole suppresses plasma estradiol and estrone sulphate more completely than anastrozole in postmenopausal women with breast cancer. J Clin Oncol 26:1671-1676

15. Miller WR, Dixon JM (2001) Local endocrine effects of aromatase inhibitors within the breast. J Steroid Biochem Mol Biol 79:93-102

16. Miller WR (1999) Biology of aromatase inhibitors: pharmacology/ endocrinology within the breast. Endocr Relat Cancer 6:187-195

17. Gershanovich M, Chaudri HA, Campos D et al (1998) Letrozole, a new oral aromatase inhibitor: randomised trial comparing $2.5 \mathrm{mg}$ daily, $0.5 \mathrm{mg}$ daily and aminoglutethimide in postmenopausal women with advanced breast cancer. Letrozole International Trial Group (AR/BC3). Ann Oncol 9:639-645

18. Nabholtz JM, Buzdar A, Pollak M et al (2000) Anastrozole is superior to tamoxifen as first-line therapy for advanced breast cancer in postmenopausal women: results of a North American multicenter randomized trial. J Clin Oncol 18:3758-3767

19. Urruticoechea A, Smith IE, Dowsett M (2005) Proliferation marker Ki-67 in early breast cancer. J Clin Oncol 23:7212-7220

20. Robertson JF, Nicholson RI, Bundred NJ et al (2001) Comparison of the short -term biological effects of 7alpha- [9- $(4,4,5,5,5$ pentafluoropentylsulfinyl)-nonyl] estra-1, 3, 5, (10)-triene-3, 17 beta-diol (Faslodex) versus tamoxifen in postmenopausal women with primary breast cancer. Cancer Res 61:6739-6746

21. Werner M, Chott A, Fabiano A et al (2000) Effect of formalin tissue fixation and processing on immunohistochemistry. Am J Surg Pathol 24:1016-1019

22. Anderson TJ, Dixon JM, Murray J et al (2004) Early changes in tumour Ki67 expression differentiate for pathological (but not clinical) response in breast cancers treated neo-adjuvantly with letrozole. Breast Cancer Res Treat 78(suppl 1):S50

23. Ellis MJ, Tao Y, Luo J et al (2007) Outcome prediction for clinical stage II and III ER+ breast cancer based on treatment response, pathological stage, tumor grade, Ki67 proliferation index, and estrogen receptor status after neoadjuvant endocrine therapy. Breast Cancer Res Treat 106(suppl 1):S16

24. Ellis MJ, Tao Y, Young O et al (2006) Estrogen independent proliferation is present in the majority of estrogen receptor positive $(E R+)$ HER2 gene amplified primary breast cancers after letrozole exposure despite frequent tumor regression during neoadjuvant treatment. J Clin Oncol 24(19):3019-3025 